\title{
Mortality reduction with levosimendan in patients with heart failure: Current evidence is underpowered
}

\author{
Filippo Sanfilippo, Luigi La Via, Federica Merola, Marinella Astuto \\ Department of Anesthesia and Intensive Care, A.O.U. Policlinico-San Marco, Catania, Italy
}

It is with great interest that we read the meta-analysis by Jaguszewski et al. [1] comparing the effects of levosimendan and dobutamine in patients with heart failure (HF). This meta-analysis is relevant and it confirms a possibly preferential role for levosimendan in this population of patients considering the significant reduction in hospital (or 30-day) mortality, as shown by the pooled analysis on the 10 included studies. This finding is not surprising, since this ino-dilator has shown a reduction in mortality for patients with severely reduced left ventricular systolic function and/or low cardiac output syndrome undergoing cardiac surgery. Moreover, levosimendan also reduced the need for renal replacement therapy after high-risk cardiac surgery [2].

However, before drawing firm conclusions on the use of levosimendan in patients with HF, an analysis of the robustness of the findings by Jaguszewski et al. [1] is needed. Therefore, it was thought that the manuscript would greatly benefit from the addition of a trial-sequential analysis (TSA), which would allow calculation of the required sample ("information size"), estimating the power of the meta-analysis on the reduction of mortality by levosimendan, as well as the need for further research.

Hereby, we would like to offer a contribution. We imported the same data provided by the authors in the TSA Software (Copenhagen Trial Unit's TSA Software ${ }^{\circledR}$; Copenhagen, Denmark). The information size was computed assuming an alpha risk of $5 \%$ with a power of $80 \%$. The estimated mortality was computed using weighted averages from the included studies (levosimendan $8.4 \%$ vs. dobuta- mine $12.6 \%$ ). We used a random effect model with mortality analyzed as odds ratio (OR). Further details on TSA and its interpretation are available elsewhere [3].

The TSA showed that current evidence is severely underpowered to determine whether levosimendan reduces mortality in patients with $\mathrm{HF}$ as compared to dobutamine. Indeed, the ratio between number of patients recruited and sample needed ( $n=2263 / 8366 ; 27 \%$; Fig. 1). Therefore, more research is certainly warranted on mortality in this population of patients.

Another minor (statistical) consideration is on the authors' choice to perform their meta-analysis using a fixed effect model, which assumes that the true effect is the same across studies. However, it is unlikely that all included studies have "identical" true effect, especially when there is statistical heterogeneity (47\% in the meta-analysis Jaguszewski et al. [1]). In such cases it is advisable to use a random effect model, which better balances the weights of the included studies [4]. For instance, moving from the fixed to the random effect model, the weight of the largest study (Mebazaa et al. [5]) on the overall results passed from $62 \%$ to $28.6 \%$. Nonetheless, our consideration does not change the meta-analysis results since levosimendan still shows significant reductions in mortality also using the random effect model (OR: $0.45 ; 95 \%$ confidence interval: $0.24-0.84 ; \mathrm{p}=0.01$ ).

In summary, in their meta-analysis Jaguszewski et al. [1] showed benefits of levosimendan over dobutamine for patients with $\mathrm{HF}$ with a significant reduction in hospital (or 30-day) mortality. However, current evidence is severely underpowered

Address for correspondence: Filippo Sanfilippo, MD, PhD, Department of Anesthesia and Intensive Care, A.O.U. Policlinico-San Marco, Catania, Italy, Via Santa Sofia, 78 - 95100 - Catania, Italy, tel: 00390953782307, fax: 0039 0953782673, e-mail: filipposanfi@yahoo.it

Received: 25.04.2021 Accepted: 3.07.2021 Early publication date: 7.07.2021

This article is available in open access under Creative Common Attribution-Non-Commercial-No Derivatives 4.0 International (CC BY-NC-ND 4.0) license, allowing to download articles and share them with others as long as they credit the authors and the publisher, but without permission to change them in any way or use them commercially. 


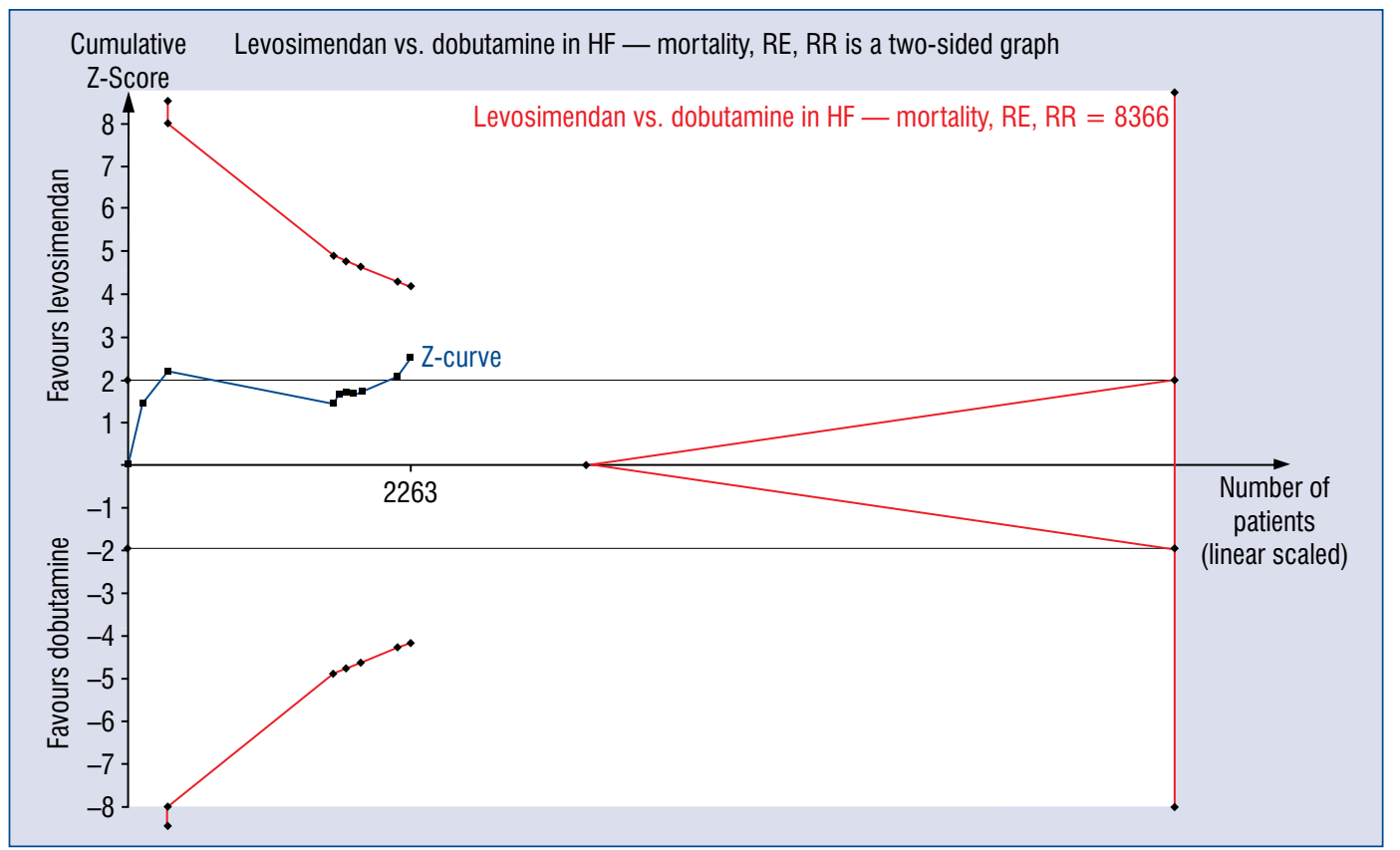

Figure 1. Trial sequential analysis on hospital (30-day) mortality in patients with heart failure (HF), comparing treatment with levosimendan versus dobutamine; RE — random effect; RR — risk reduction.

and further randomized research is required before drawing firm conclusions.

\section{Conflict of interest: None declared}

\section{References}

1. Jaguszewski MJ, Gasecka A, Targonski R, et al. Efficacy and safety of levosimendan and dobutamine in heart failure: A systematic review and meta-analysis. Cardiol J. 2021; 28(3): 492-493, doi: 10.5603/CJ.a2021.0037, indexed in Pubmed: 33843036.

2. Sanfilippo F, Knight JB, Scolletta S, et al. Levosimendan for patients with severely reduced left ventricular systolic function and/or low cardiac output syndrome undergoing cardiac sur- gery: a systematic review and meta-analysis. Crit Care. 2017; 21(1): 252, doi: 10.1186/s13054-017-1849-0, indexed in Pubmed: 29047417.

3. Afshari A, Wetterslev J. When may systematic reviews and metaanalyses be considered reliable? Eur J Anaesthesiol. 2015; 32(2): 85-87, doi: 10.1097/EJA.0000000000000186, indexed in Pubmed: 25536187.

4. Barili F, Parolari A, Kappetein PA, et al. Statistical Primer: heterogeneity, random- or fixed-effects model analyses? Interact Cardiovasc Thorac Surg. 2018; 27(3): 317-321, doi: 10.1093/ icvts/ivy163, indexed in Pubmed: 29868857.

5. Mebazaa A, Nieminen MS, Packer M, et al. Levosimendan vs dobutamine for patients with acute decompensated heart failure: the SURVIVE Randomized Trial. JAMA. 2007; 297(17): 1883-1891, doi: 10.1001/jama.297.17.1883, indexed in Pubmed: 17473298. 
